\title{
MicroRNA-21 regulates the expression of BTG2 in HepG2 liver cancer cells
}

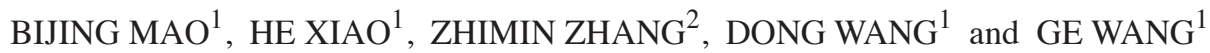 \\ ${ }^{1}$ Cancer Center, Institute of Surgical Research, Daping Hospital, Third Military Medical University, \\ Chongqing 400042; ${ }^{2}$ Department of Oncology, Wuhan General Hospital of Guangzhou Command, \\ People's Liberation Army, Wuhan, Hubei 430070, P.R. China
}

Received September 24, 2014; Accepted June 11, 2015

DOI: $10.3892 / \mathrm{mmr} .2015 .4051$

\begin{abstract}
B-cell translocation gene 2 (BTG2) is a tumor suppressor gene, which belongs to the anti-proliferation gene family. Our previous study demonstrated that microRNA (miR)-21 and the expression of BTG2 were negatively correlated during hepatocarcinogenesis. The aim of the present study was to investigate the effects of miR-21 on the growth and progression of liver cancer cells, and to determine the underlying mechanism. A luciferase reporter assay was used to demonstrate that the BTG2 gene was a direct target of miR-21. In addition, the effects of miR-21 on cell growth and gene expression in HepG2 human hepatocellular carcinoma (HCC) cells were analyzed using reverse transcription-quantitative polymerase chain reaction, western blotting, an MTT assay, flow cytometry, a Transwell invasion assay and a wound healing assay. The expression levels of miR-21 in the HepG2 cells were significantly higher, compared with those in L02 normal liver cells. The expression levels of BTG2 in liver cancer cell lines (HepG2 and Huh7) were significantly lower, compared with that in the L02 cells. These results suggested that BTG2 was the direct target gene of miR-21. The protein expression levels of BTG2 were inhibited by high expression levels of miR-21, and increased by inhibition of the expression of miR-21 in the HepG2 cells. Inhibition of miR-21 reduced cell proliferation and invasion, and increased the rate of apoptosis in the HepG2 cells. These results indicated that miR-21 regulates cell proliferation, invasion, migration and apoptosis in HepG2 cells, which may be associated with its effects on the expression of BTG2. The results of the present study may provide a basis for targeting the miR-21/BTG2 interaction for the treatment of HCC.
\end{abstract}

Correspondence to: Dr Ge Wang, Cancer Center, Institute of Surgical Research, Daping Hospital, Third Military Medical University, 10 Changjiang Road, Chongqing 400042, P.R. China E-mail: wangge70@hotmail.com

Key words: human hepatocellular carcinoma, microRNA-21, B-cell translocation gene 2 , proliferation, apoptosis, invasion, migration

\section{Introduction}

Hepatocellular carcinoma (HCC) is the most common type of liver cancer, which accounts for $~ 85-90 \%$ of all types of primary liver cancer, and is one of the most life-threatening forms of cancer (1). Despite marked progress in the treatment of HCC, the survival rate remains poor and the molecular pathogenesis of HCC remains to be fully elucidated. The development and progression of $\mathrm{HCC}$ is a complex process, which involves the dysregulation of oncogenes and tumor suppressor genes. It has previously been reported that microRNAs (miRNAs) are essential in oncogenesis by the regulation of oncogenes and tumor suppressor genes (2-4). Previous studies have reported a link between the aberrant expression of miRNAs and malignant tumor development, including HCC (5-8), increasing understanding of the underlying molecular mechanisms and novel therapeutic targets of HCC.

miRNAs are widely distributed, non-coding, small RNAs, which regulate target gene expression through binding to the 3'-untranslated regions (3'UTRs) of target mRNAs at the translational level (9). It has previously been reported that $\leq 30 \%$ of human genes are regulated by miRNAs (10). miRNAs have been suggested to be biomarkers for HCC diagnosis (11). Among those miRNAs, miR-21 was one of the first to be discovered, and is considered one of the most important miRNAs, which is overexpressed in various types of carcinomas, including prostate, gastric, colon, breast and lung cancer (12-17). As a biomarker, miR-21 has a higher sensitivity, compared with carcinoembryonic antigen and cancer antigen 15-3 in the diagnosis of breast cancer $(18,19)$. In colorectal cancer, miR-21 has been suggested as a potential biomarker for diagnosis and as a target for therapy (20) and radiotherapy (21). In addition, miR-21 has been observed to stimulate gastric cancer growth and invasion through the inhibition of phosphatase and tensin homolog (PTEN) and programmed cell death protein 4 (PDCD4) (22). The inhibition of miR-21 decreases the proliferation and invasion of lung cancer cells by increasing the expression of PTEN (17), indicating that PTEN is a direct target gene of miR-21.

Previous studies have demonstrated that B-cell translocation gene 2 (BTG2) is regulated by miR-21 in laryngeal cancer and prostate cancer cells $(13,23)$. BTG2 was the first gene to be identified in the BTG/Tob anti-proliferation gene 
family (24). BTG2 is expressed in the majority of normal tissues, with high levels being detected in the lungs, kidneys, intestines, pancreas, and prostate (25). BTG2 is an instantaneous early response protein, which is involved in cell differentiation, anti-proliferation, DNA damage repair and cell apoptosis (26-29). In addition, our previous study demonstrated that p53 positively regulates BTG2 and negatively regulates cyclin D1 and cyclin E, indicating a critical role in hepatocarcinogenesis $(30,31)$. The expression of BTG2 is reduced or absent in various types of cancer, including melanoma, breast, gastric and lung cancers (26,32-34). It has also been suggested that BTG2 is regulated by miR-21 in laryngeal and prostate cancer cells $(13,23,34)$ However, whether low expression levels of BTG2 are associated with high expression levels of miRNA-21 in liver cancer cells remains to be elucidated. The aim of the present study was to investigate the mechanism by which BTG2 is regulated by miR-21, and determine the role of miR-21 in the growth and progression of HCC.

\section{Materials and methods}

Cell lines. The Hep3B, Huh-7, QGY7701 and HepG2 human HCC cell lines, and the L02 normal human liver cell line, were obtained from the Liver Cancer Institute of Zhongshan Hospital, Fudan University (Shanghai, China). The cells were cultured in Dulbecco's modified Eagle's medium (DMEM) supplemented with $10 \%$ fetal bovine serum (FBS; Invitrogen Life Technologies, Carlsbad, CA, USA) at $37^{\circ} \mathrm{C}$ in a humidified incubator containing $5 \% \mathrm{CO}_{2}$. The media were replaced every other day and cells were passaged every 2 or 3 days once they had reached $70-80 \%$ confluence. The cells in the exponential growth phase were harvested and used for the subsequent experiments.

Cell transfection. An miR-21 inhibitor, anti-miR-21 (sequence, 5'-UCAACAUCAGUCUGAUAAGCUA-3'); which is a chemically modified single strand RNA that is a competitive inhibitor of miR-21, and a mismatched negative control (miR-21 inhibitor NC) were obtained from Shanghai GenePharma Co., Ltd. (Shanghai, China). Lipofectamine ${ }^{\circledR} 2000$ was purchased from Invitrogen Life Technologies. Transfection of the cells with anti-miR-21 or NC was performed using Lipofectamine ${ }^{\circledR} 2000$ in Opti-MEM, according to the manufacturer's instructions. Briefly, diluted Lipofectamine ${ }^{\circledR} 2000(3.75 \mu \mathrm{l})$ was mixed with diluted miR-21 inhibitor or NC (150 nmol/l), and incubated at room temperature for $20 \mathrm{~min}$ with gentle agitation. The mixture was then added to the HepG 2 cell culture plates $(60 \%$ confluent), and incubated at $37^{\circ} \mathrm{C}$ in an atmosphere containing $5 \% \mathrm{CO}_{2}$. Following a $6 \mathrm{~h}$ incubation, the medium was replaced with fresh medium, supplemented with $10 \% \mathrm{FBS}$, and the cells were cultured for a further $48 \mathrm{~h}$ prior to performing the subsequent experiments.

Luciferase-reporterassay. TargetgenesofmiR-21 werepredicted by PicTar (http://pictar.mdc-berlin.de/cgi-bin/PicTar_vertebrate.cgi) and Targetscan (http://www.targetscan.org/). The HepG2 cells (Changsha-run Win Biotechnology, Inc., Hunan, China) were co-transfected with a pYr-MirTarget-BTG2-3U plasmid (0.8 $\mu \mathrm{g}$; Changsha-run Win Biotechnology, Inc.) and either the miRNA NC (75 nmol/1), miR-21 mimics (75 nmol/l; sequence, 5'-UAGCUUAUCAGACUGAUGUUGAAAC AUCAGUCUGAUAAGCUAUU-3'; Shanghai GenePharma Co., Ltd.) or miR-21 inhibitor (150 nmol/l; Shanghai GenePharma Co., Ltd.). In addition, HepG2 cells were transfected with miR-21 mimiwes and pYr-Mir Target plasmid, pYr-Mir Target-BTG2-3U plasmid, or pYr-Mir Target-BTG2-3U-Delete site plasmid (Changsha-run Win Biotechnology, Inc.). Transfection was performed using Lipofectamine ${ }^{\circledR} 2000$, on $60 \%$ confluent cells seeded in 24-well plates, at $37^{\circ} \mathrm{C}$ for $48 \mathrm{~h}$. At $24 \mathrm{~h}$ post-transfection, the activities of firefly and Renilla luciferase were measured using a Dual-Luciferase Reporter assay (Promega Corporation, Madison, WI, USA). The activity of Renilla luciferase for each sample was normalized to that of firefly luciferase. Each experiment was repeated twice and assessed in triplicate.

Western blotting. The cells were lysed and the total protein was extracted using radioimmunoprecipitation (RIPA) buffer, containing $150 \mathrm{mM} \mathrm{NaC1}, 25 \mathrm{mM}$ Tris-HCl (pH 7.6), 1\% sodium deoxycholate, $0.1 \%$ SDS and 1\% NP-40 (Thermo Fisher Scientific, Inc., Rockford, IL, USA). Protease inhibitor (cat. no. 78410; Thermo Fisher Scientific, Inc.) was added to the RIPA buffer prior to use. Total protein concentrations were quantified using the Bicinchoninic Acid Protein Assay kit (Beyotime Institute of Biotechnology, Shanghai. China). Samples with equal quantities of total protein $(100 \mu \mathrm{g})$ were fractionated by $12 \%$ SDS-PAGE and transferred onto nitrocellulose membranes (Bio-Rad Laboratories, Inc., Hercules, CA, USA). The expression levels of BTG2 were probed using primary monoclonal mouse anti-human BTG2 (1:500; cat. no. ab58219; Abcam, Cambridge, MA, USA), and the internal control $\beta$-actin was detected using primary monoclonal mouse anti-human $\beta$-actin (1:1,000; cat. no. sc-47778; Santa Cruz Biotechnology, Inc., Dallas, TX, USA). The secondary antibody used was goat anti-mouse $\operatorname{IgG}(\mathrm{H}+\mathrm{L}), \mathrm{HRP}$ conjugate (1:5,000; cat. no. 31430; Thermofisher Technology Co., Ltd, Shanghai, China). The signals of the bands were visualized using an enhanced chemiluminescence method (Thermo Fisher Scientific, Inc.). Protein expression levels were quantified using a Gel Documentation system (Gel Doc ${ }^{\mathrm{TM}} \mathrm{EQ}$; cat. no. 170-8060; Bio-Rad Laboratories, Inc.).

RNA extraction and reverse transcription-quantitative polymerase chain reaction $(R T-q P C R)$. Total RNA was extracted from the cells using TRIzol ${ }^{\circledR}$ reagent (Invitrogen Life Technologies), according to the manufacturer's instructions. Reverse transcription was conducted using RevertAid Reverse Transcriptase (Shanghai GenePharma Co., Ltd). Quantification of mature miRNAs was performed using a Quantitect SYBR Green PCR kit (Changsha-run Win Biotechnology, Inc.) and a 7500 Multiplex Quantitative PCR system (Applied Biosystems Life Technologies, Foster City, CA, USA), according to the manufacturer's instructions. The PCR reaction mix consisted of: $5 \mu 12 X$ PCR Master mix, $0.3 \mu \mathrm{l}$ forward primer $(10 \mu \mathrm{M}), 0.3 \mu \mathrm{l}$ reverse primer $(10 \mu \mathrm{M})$, $2 \mu \mathrm{l}$ cDNA, and $2.4 \mu \mathrm{l} \mathrm{H}_{2} \mathrm{O}$. The PCR cycling conditions were as follows: $95^{\circ} \mathrm{C}$ for $5 \mathrm{~min}$, followed by 42 cycles at $95^{\circ} \mathrm{C}$ for $30 \mathrm{sec}, 59^{\circ} \mathrm{C}$ for $30 \mathrm{sec}$ and $72^{\circ} \mathrm{C}$ for $20 \mathrm{sec}$, and $95^{\circ} \mathrm{C}$ for 10 sec. The U6 gene was used as an internal control and each reaction was performed in triplicate. The primer (Shanghai 
GenePharma Co., Ltd) sequences were as follows: miR-21, forward 5'-GCGGCGTAGCTTATCAGACTGA-3', reverse 5'-GTGCAGGGTCCGAGGT-3'; and U6, forward 5'-CTC GCTTCGGCAGCACA-3', and reverse 5'-AACGCTTC ACGAATTTGCGT-3'. The fold-change of miR-21 expression in HepG2 cells was compared with that in the L02 normal liver cells using the $2^{-\Delta \Delta C T}$ method (35).

Measurement of cell proliferation. An MTT assay was used to detect cell proliferation. Briefly, the cells were made into a single cell suspension using DMEM, supplemented with $10 \%$ FBS, and seeded into 96 -well plates at a density of $1 \times 10^{4}$ cells in $200 \mu 1 /$ well. The cells were then cultured for $24 \mathrm{~h}$ at $37^{\circ} \mathrm{C}$, to allow them to adhere to the plate. Subsequently, $20 \mu 1$ MTT solution $(5 \mathrm{mg} / \mathrm{ml}$; Beyotime Institute of Biotechnology) was added to each well and incubated for $4 \mathrm{~h}$ at $37^{\circ} \mathrm{C}$. The culture medium was then discarded, and $150 \mu \mathrm{l}$ DMSO (Sigma-Aldrich, St. Louis, MO, USA) was added to each well, and the plates were agitated for $15 \mathrm{~min}$ in order to let the crystalized precipitates fully dissolved. The optical density values were measured at $490 \mathrm{~nm}$ using an ELISA monitor (Multiskan MK3; Thermo Fisher Scientific). Each experimental group contained nine replicate wells, and the experiment was performed in triplicate.

Detection of cell migration using a scratch wound assay. Once the cells had reached $70 \sim 80 \%$ confluence, a wound (1-1.5 mm wide) was made using a sterile tip. The wells were gently washed three times with PBS. The cells were subsequently cultured in serum-free DMEM at $37^{\circ} \mathrm{C}$. At 0 , 24,30 and $48 \mathrm{~h}$ post-wounding, the distance of the scratch between the cells was evaluated under an Olympus inverted microscope (CKX41; Olympus Corporation, Tokyo, Japan).

Measurement of cell invasion using a Transwell invasion assay. A Transwell invasion assay was used to detect the invasive ability of the treated HepG2 cells and control cells. The cells $\left(1 \times 10^{5}\right.$ cells suspended in $300 \mu 1$ serum-free DMEM) were seeded onto the upper chamber of a Transwell invasion system (Changsha-run Win Biotechnology, Inc.), and $500 \mu \mathrm{l}$ DMEM supplemented with 10\% FBS was added to the lower chamber. The chamber was then incubated at $37^{\circ} \mathrm{C}$ in a humidified incubator containing $5 \% \mathrm{CO}_{2}$ for $24 \mathrm{~h}$. The cells on the upper surface of the base membrane were removed using a sterile cotton ball. The cells, which had invaded to the lower surface of the base membrane were stained with $1 \%$ crystal violet (Changsha-run Win Biotechnology, Inc.). The cells that had successfully crossed the Transwell polycarbonate membrane were counted under an Olympus inverted microscope (Olympus Corporation). A total of of five fields were randomly selected for the calculation of each sample, and the experiment was repeated at least three times. The invasive ability of the cells was determined by the number of cells that crossed the Transwell polycarbonate membrane.

Analysis of cell cycle distribution. Once the cells had reached $70 \sim 80 \%$ confluence, the medium was replaced with serum-free medium. The cells were cultured for $48 \mathrm{~h}$, in order to reach synchronization. The treated cells were then digested, fixed with anhydrous alcohol overnight and stained with propidium
A

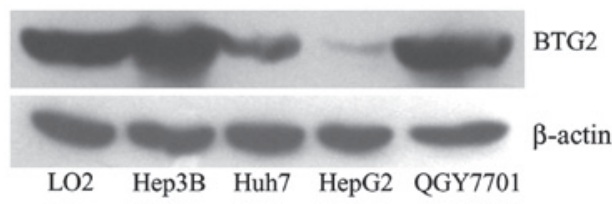

B

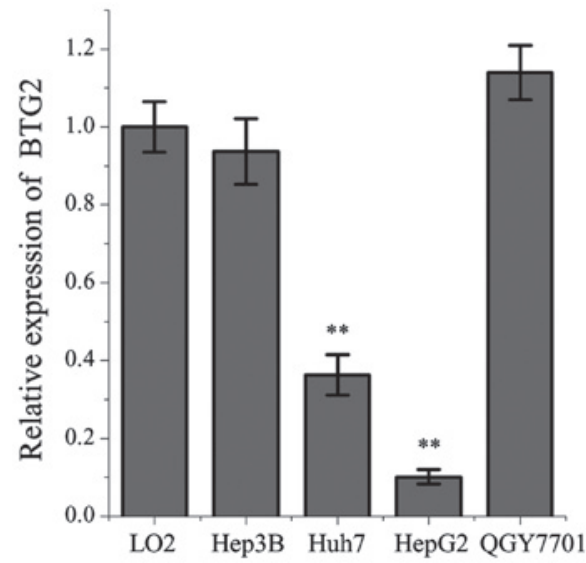

Figure 1. Protein expression levels of BTG2 in the Hep3B, Huh-7, QGY-7701 and HepG2 liver cancer cell lines and in L02 normal liver cells. (A) Representative western blots. (B) Quantitative analysis of three experiments. ${ }^{* *} \mathrm{P}<0.01$, compared with the L02 cells. Data are presented as the mean \pm standard deviation. BTG2, B-cell translocation gene 2 .

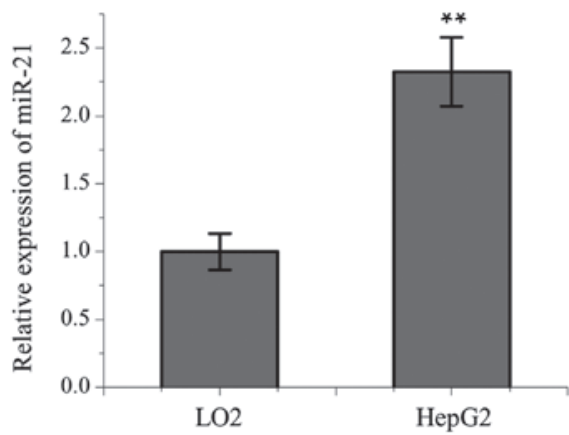

Figure 2. Comparison of the expression levels of miR-21 between HepG2 liver cancer cells and L02 normal liver cells. ${ }^{* *} \mathrm{P}<0.01$, compared with the L02 cells. Data are presented as the mean \pm standard deviation. miR-21, microRNA-21.

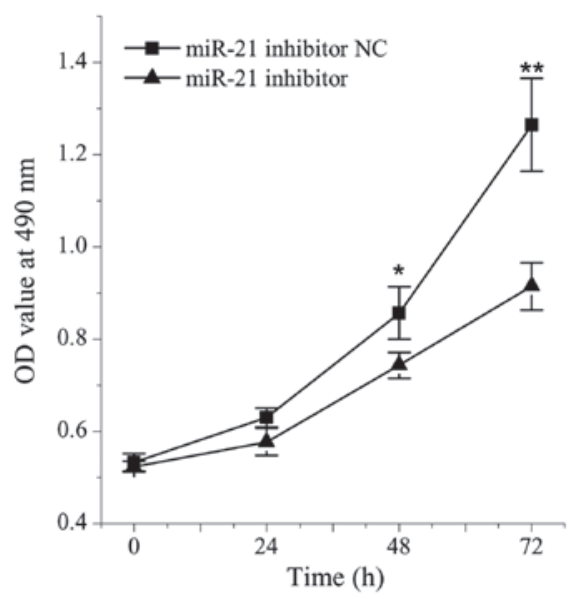

Figure 3. Effects of miR-21 inhibitor on the viability of HepG2 liver cancer cells. ${ }^{*} \mathrm{P}<0.05,{ }^{* *} \mathrm{P}<0.01$, compared with the NC-transfected cells. Data are presented as the mean \pm standard deviation. miR-21, microRNA-21; NC, negative control; OD, optical density. 
iodide (100 $\mu \mathrm{g} / \mathrm{ml}$; Changsha-run Win Biotechnology, Inc.) for $30 \mathrm{~min}$ in the dark. The cell cycle distribution was detected by flow cytometry (FACSCalibur; BD Biosciences, Franklin Lakes, NJ, USA). Each experiment was repeated in triplicate and the average values of each cell cycle phase were calculated.

Analysis of apoptosis. The HepG2 cells $\left(1-5 \times 10^{5}\right)$ were harvested and gently re-suspended in $500 \mu \mathrm{l}$ binding buffer (Nanjing KeyGEN Biotech. Co., Ltd., Nanjing, China), to which $5 \mu 1$ annexin V-allophycocyaninand $5 \mu 1$ 7-aminoactinomycin D (Nanjing KeyGEN Biotech. Co., Ltd.) were added. The cells were maintained at room temperature for $15 \mathrm{~min}$ in the dark and then analyzed using flow cytometry (BD Biosciences). Each experiment was performed in triplicate.

Statistical analysis. All data were obtained from at least three independent experiments and are presented as the mean \pm standard deviation. SPSS 20.0 statistical analysis software (IBM SPSS, Armonk, NY, USA) was used for data analysis. Student's t test was used to compare data between two groups. $\mathrm{P}<0.05$ was considered to indicate a statistically significant difference.

\section{Results}

Expression levels of BTG2 and miR-21 in HCC cells. The expression levels of BTG2 were significantly lower in the Huh-7 and HepG2 HCC cells, compared with in the L02 normal human liver cell line (Fig. 1). Among the cancer cell lines, the expression levels of BTG2 were lowest in the HepG2 cells. The expression levels of miR-21 were significantly higher in the HepG2 cells, compared with in the L02 cells (Fig. 2).

Effects of miR-21 on the growth of HepG2 cells. The present study aimed to further analyze the association between miR-21 and cell growth by comparing the cell viabilities between cells treated with miR-21 inhibitor and NC. The viability of the HepG2 cells transfected with the miR-21 inhibitor was significantly lower, compared with the cells transfected with the NC (P<0.05; Fig. 3).

Effect of miR-21 inhibition on cell cycle distribution in HepG2 cells. Compared with the NC-transfected cells, the mean number of cells in the $\mathrm{G}_{2}$ phase was significantly higher, and the mean number of cells in the $\mathrm{S}$ phase was significantly lower, in the HepG2 cells transfected with the miR-21 inhibitor (all $\mathrm{P}<0.05$; Fig. 4). These results indicated that miR-21 facilitated cell cycle progression through the $\mathrm{G}_{2}$ phase.

Effects of miR-21 inhibition on the migration and invasion of HepG2 cells. Cell migration was significantly inhibited following transfection of the HepG2 cells with the miR-21 inhibitor, measured by the distance of the scratch between the cells and the rate of migration, compared with the NC-transfected cells $(\mathrm{P}<0.05$; Fig. 5A). In addition, the number of HepG2 cells transfected with the miR-21 inhibitor that crossed the polycarbonate membrane of the Transwell invasion chamber was significantly lower, compared with the NC-transfected cells ( $\mathrm{P}<0.01$; Fig. 5B).
A
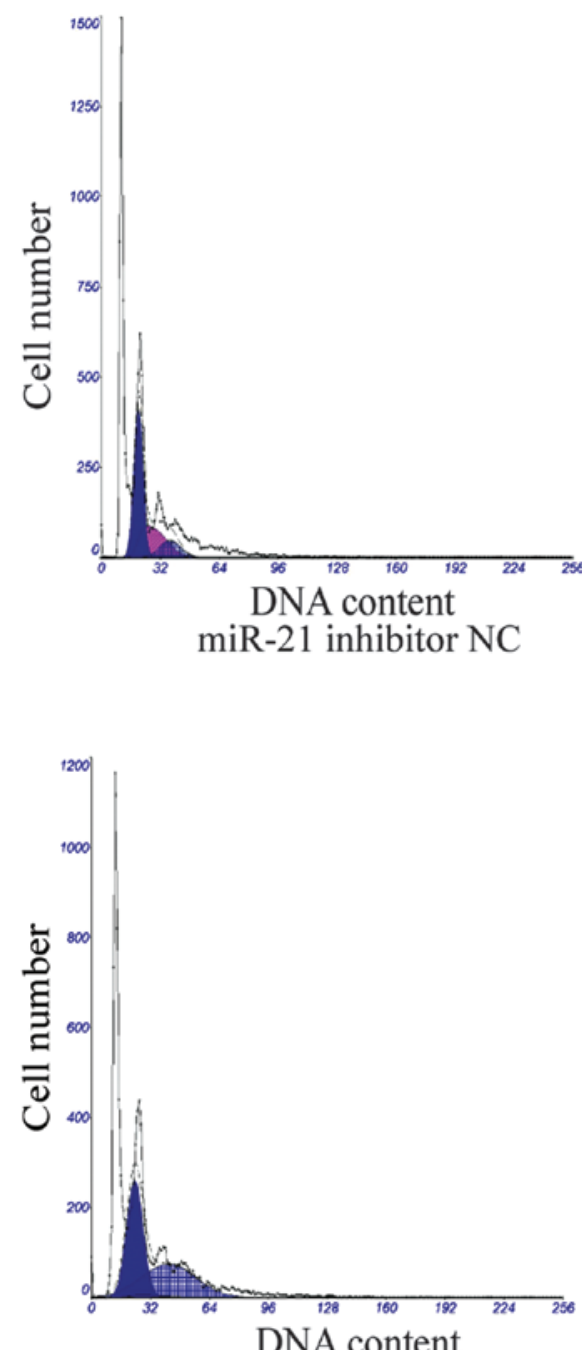

DNA content

miR-21 inhibitor

B

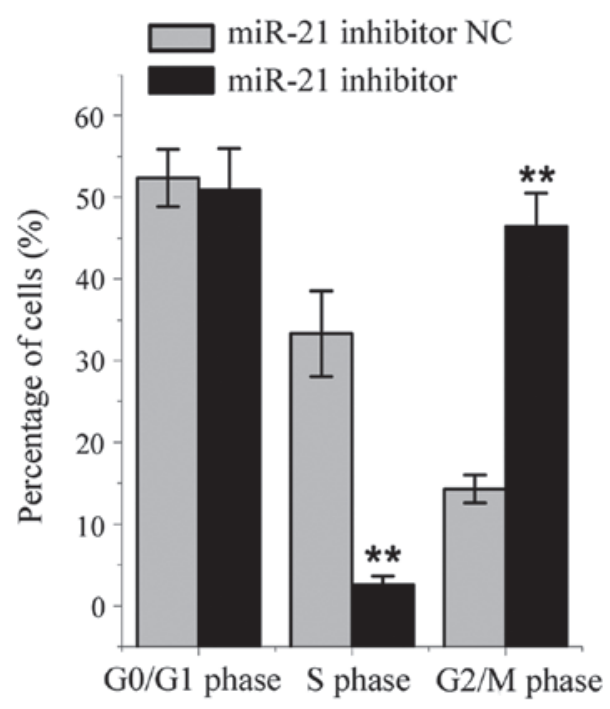

Figure 4. Effects of miR-21 inhibition on the cell cycle distribution of HepG2 liver cancer cells. (A) Representative data of the flow cytometric analysis; (B) Mean percentages of cells in each phase of the cell cycle. ${ }^{* * *} \mathrm{P}<0.01$, compared with the NC-transfected cells. Data are presented as the mean \pm standard deviation. miR-21, microRNA-21; NC, negative control. 
A
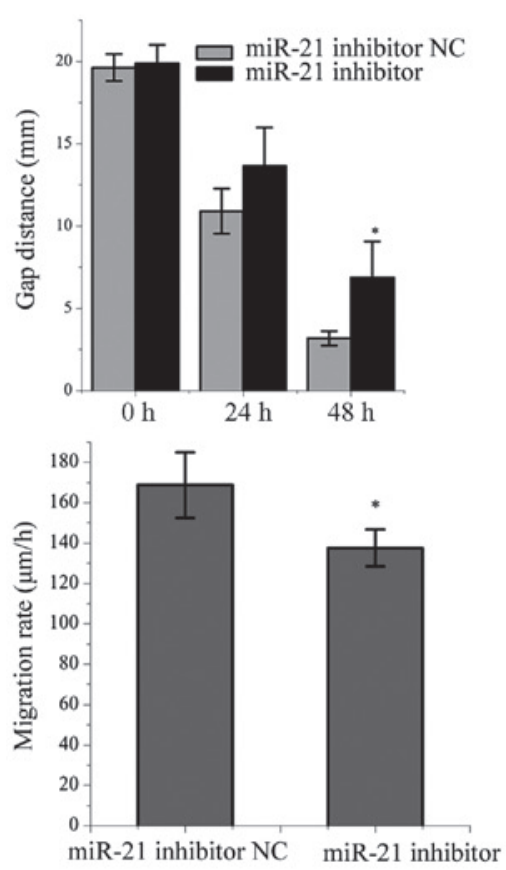

B

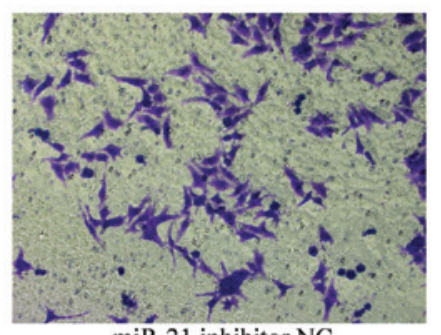

miR-21 inhibitor NC

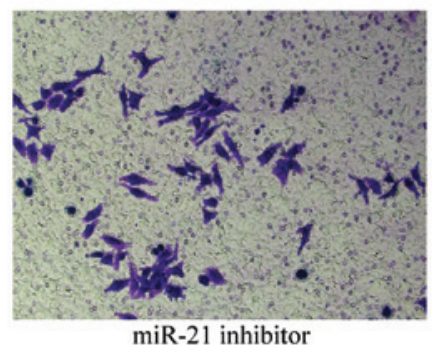

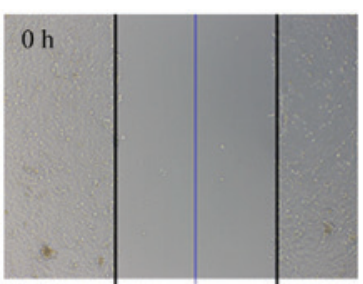
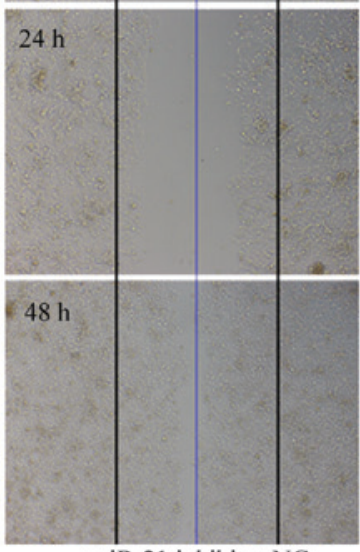

miR-21 inhibitor $\mathrm{NC}$
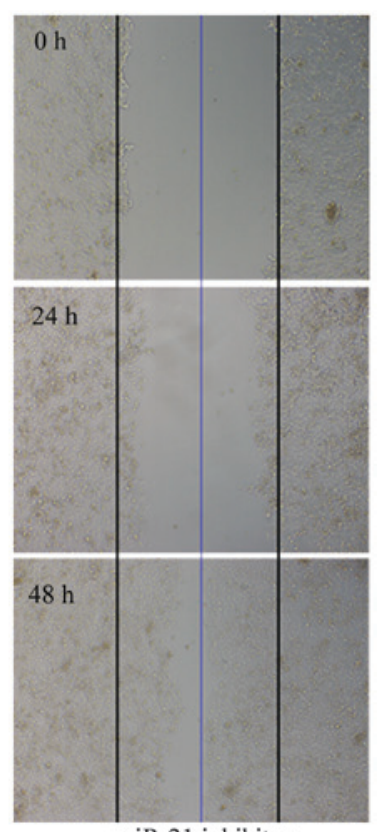

miR-21 inhibitor

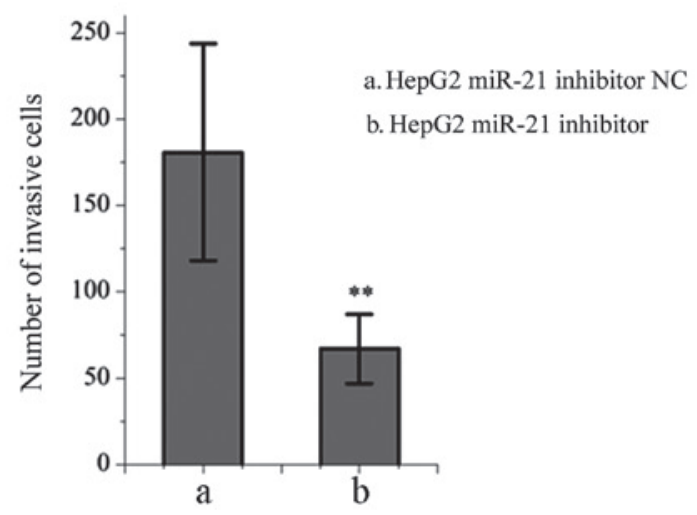

Figure 5. Effects of miR-21 inhibition on the migration and invasion of HepG2 liver cancer cells. (A) Comparison of the migration distance/migration rate of HepG2 cells treated with miR-21 inhibitor or miR-21 inhibitor NC. (B) Comparison of the number of HepG2 cells treated with the (a) miR 21 inhibitor or (b) miR-21 inhibitor $\mathrm{NC}$ that crossed the polycarbonate membrane of the transwell invasion chamber (magnification, $\mathrm{x} 200$ ). ${ }^{*} \mathrm{P}<0.05,{ }^{* *} \mathrm{P}<0.0001, \mathrm{compared}$ with the NC-transfected cells. Data are presented as the mean \pm standard deviation. miR-21, microRNA-21; NC, negative control.

A

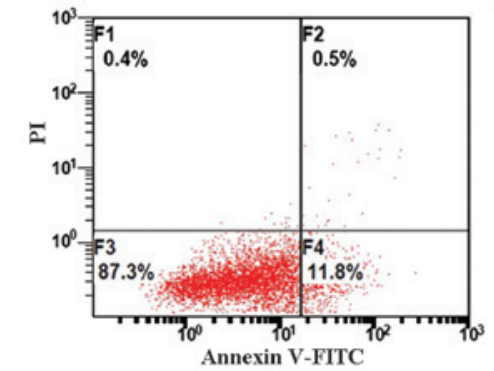

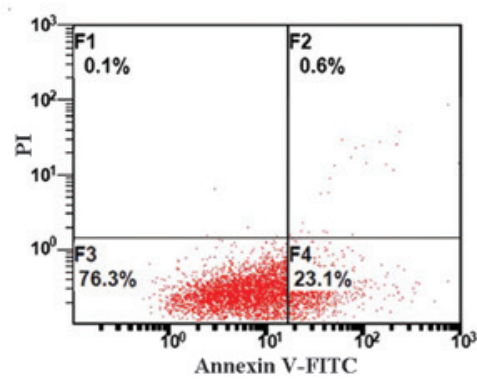

B

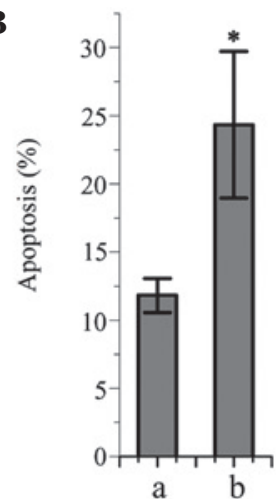

a) HepG2 miR-21 inhibitor $\mathrm{NC}$

b) HepG2 miR-21 inhibitor

Figure 6. Effects of miR-21 inhibitor on the apoptosis of HepG2 liver cancer cells. (A) Representative flow cytometric analysis; (B) Relative percentages of apoptotic cells following transfection with (a) miR-21 inhibitor $\mathrm{NC}$ or (b) miR-21 inhibitor. "P<0.05, compared with the NC-transfected cells. miR-21, microRNA-21; NC, negative control; FITC, fluorescein isothiocyanate; PI, propidium iodide. 
A

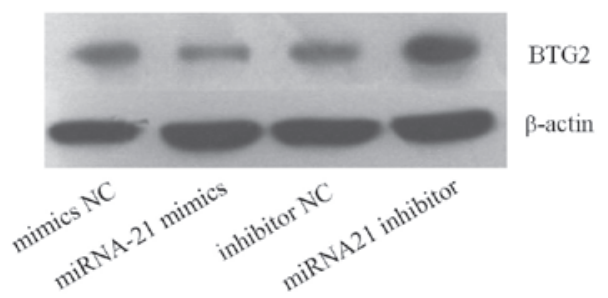

B

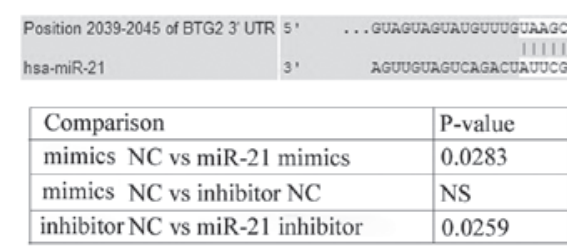

C

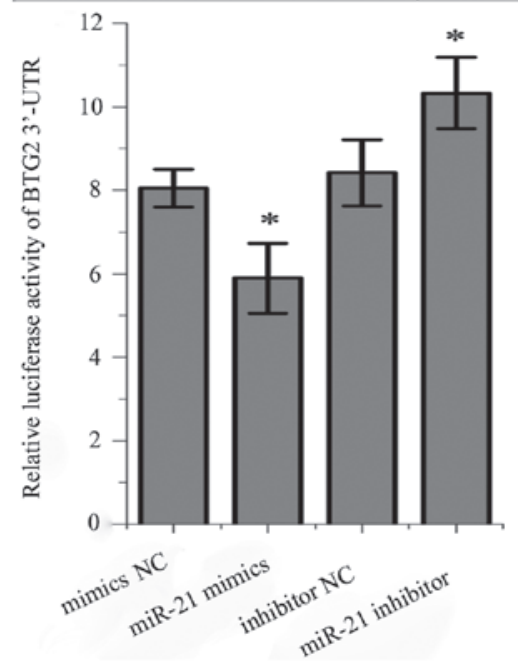

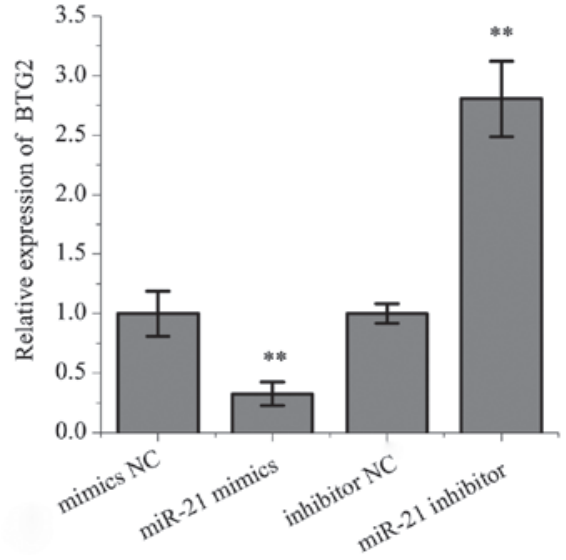

D

\begin{tabular}{|l|l|}
\hline Comparison & P-value \\
\hline Vector vs Wild type & $<0.01$ \\
\hline Mutation vs Wild type & 0.0355 \\
\hline
\end{tabular}

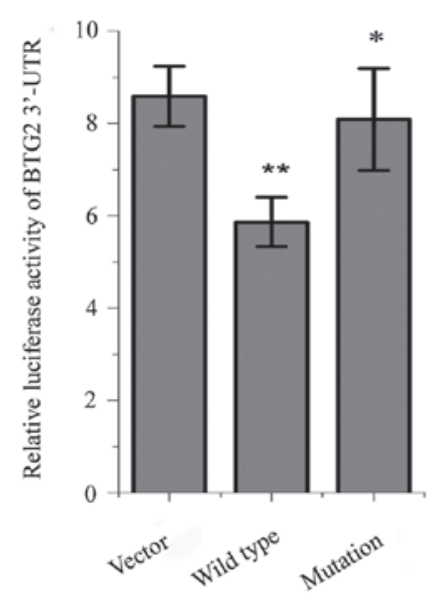

Figure 7. BTG2 is a direct target gene of miR-21. (A) Protein expression levels of BTG2 were increased in HepG2 liver cancer cells transfected with the miR-21 inhibitor, compared with those transfected with the miR-21 inhibitor NC. The protein expression levels of BTG2 decreased in the HepG2 cells transfected with miR-21 mimics, compared with those transfected with the NC. (B) Position of the 3'UTR of BTG2 binding with miR-21. (C) Luciferase activity was significantly decreased in the HepG2 cells following co-ntransfection with the pYr-MirTarget-BTG2-3'U plasmid and miR-21 mimics, but was increased following co-transfection with the pYr-MirTarget-BTG2-3'U plasmid and miR-21 inhibitor, compared with the NC group. (D) Following deletion of the predicted binding site of BTG2 3'UTR, the HepG2 cells were co-transfected with miR-21 mimics and a pYr-MirTarget plasmid (vector), pYr-MirTarget-BTG2-3U plasmid (wild type) or pYr-MirTarget-BTG2-3U-Delete site plasmid (mutation). The results revealed that the luciferase activities were significantly enhanced following deletion of the predicted binding site, compared with the wild-type (mimic) group. ${ }^{*} \mathrm{P}<0.05,{ }^{* *} \mathrm{P}<0.01$, the mimics NC group compared with miR-21 mimics, and the inhibitor group compared with miR-21 inhibitor group. BTG2, B-cell translocation gene 2; miR-21, microRNA-21; NC, negative control; UTR, untranslated region.

Effects of miR-21 inhibition on the apoptosis of HepG2 cells. The number of apoptotic cells in the miR-21 inhibitor-transfected group was significantly higher, compared with number in the NC-transfected group $(\mathrm{P}<0.05$; Fig. 6$)$. These results suggested that miR-21 may prevent apoptosis in HepG2 cells.

BTG2 as a direct target gene of miR-21 in HepG2 cells. According to publicly available databases, including TargetScan and PicTar $(36,37)$, the BTG2 gene is a predicted target gene of miR-21. As shown in Fig. 7A, inhibition of the expression of miR-21 promoted the protein expression of BTG2 in HepG2 cells, compared with the NC-transfected cells. In the luciferase reporter assay, the signal in the HepG2 cells, which were co-transfected with the BTG2-3'-UTR plasmid and miR-21 mimic was significantly decreased, whereas the luciferase signal was increased in the cells co-transfected with the
BTG2-3'-UTR plasmid and miR-21 inhibitor (Fig. 7B and C). Following deletion of the predicted binding site of the BTG2 3'UTR, theHepG2 cells were co-transfected with miR-21 mimics and pYr-MirTarget plasmid, pYr-MirTarget-BTG2-3U plasmid, or pYr-MirTarget-BTG2-3U-Delete site plasmid. As shown in Fig. 7D, the luciferase activities were significantly enhanced following deletion of the predicted binding site, compared with the wild-type group, suggesting that BTG 2 is a direct target gene of miR-21.

\section{Discussion}

As a novel class of regulatory molecules, miRNAs are important in cancer development and progression. It has been reported that almost half of miRNAs are located in cancer-associated gene regions and vulnerable regions $(38,39)$. 
The present study aimed to determine the roles of miRNAs in liver carcinogenesis. Our previous studies demonstrated that miR-224 is significantly upregulated in HepG2 cells, and is able to regulate cell migration and invasion via the homeobox D10/phosphorylated-PAK4/matrix metalloproteinase (MMP)-9 signaling pathway $(40,41)$. In the present study, inhibition of miR-21 suppressed cell proliferation, induced $\mathrm{G}_{2} / \mathrm{M}$ phase arrest, inhibited cell migration and promoted apoptosis in the HepG2 cells.

miRNAs regulate cellular biological functions by affecting the expression of several target genes. It has previously been reported that miR-21 has various target genes, including PTEN, PDCD4 and tropomyosin $1(22,42)$. BTG2 has also been suggested as a target gene of miR-21 in laryngeal and prostate cancer cells $(13,23)$. In the present study, it was demonstrated that BTG2 was a target gene of miR-21. Firstly, the BTG2 gene was predicted to be a target gene of miR-21 following database searches, including TargetScan and PicTar, which indicated that the 3'UTR of BTG2 included a sequence matching the miR-21 seed region. Secondly, the protein expression levels of BTG2 were negatively correlated with the expression levels of miR-21 in the HepG 2 cells. Thirdly, the overexpression of miR-21 decreased the luciferase activity in the HepG2 cells, whereas inhibition of miR-21 enhanced the luciferase signal. Finally, a deletion mutation in the BTG2 3'UTR affected the regulatory effects of miR-21.

It has been reported that overexpression of BTG2 inhibits the expression of several genes in lung cancer cells, including cyclin D1, MMP-1 and MMP-2 (26). In addition, BTG2 suppresses the growth and proliferation of gastric cancer cells (34). BTG2 promotes cell apoptosis and inhibits cancer cell invasion (29), however, Wagener et al (27) reported that endogenous expression of BTG2 contributes to the migration of bladder cancer cells. The results of the present study demonstrated that the expression of BTG2 was downregulated in hepatocarcinoma cells, indicating that the loss of BTG2 may promote carcinogenesis. The overexpression of miR-21 may contribute to the downregulated expression of BTG2 in hepatocarcinoma, however, alternative molecular mechanisms cannot be ruled out.

BTG2 acts as a cell cycle regulatory molecule and is important in inducing $\mathrm{G}_{1} / \mathrm{S}$ arrest through downregulation of cyclin D1. The activation of BTG2 depends on p53 (24). However, in the present study, suppression of the expression of miR-21 prevented cell cycle progression via inducing $G_{2} / M$ phase arrest, and no significant effect on $\mathrm{G}_{1}$ phase was observed. It has previously been reported that BTG2 induces $\mathrm{G}_{2} / \mathrm{M}$ arrest and cell death by inhibiting cyclin B1-Cdc2 binding $(24,43)$. Therefore, it was hypothesized that inhibition of cell proliferation and growth partly depend on BTG2-induced $\mathrm{G}_{2} / \mathrm{M}$ phase arrest, through inhibition of cyclin B1-Cdc2 binding. Further investigations are required to understand the underlying molecular mechanisms.

In conclusion, the results of the present study suggested that miR-21 may be important in the development and progression of hepatocarcinoma. In the present study, miR-21 was found to be overexpressed and BTG2 was found to to be downregulated in HepG2 liver cancer cells. Inhibition of miR-21 suppressed cell proliferation, migration and invasion, and promoted cell apoptosis. In addition, the inhibition of cell growth partly depended on BTG2-induced $\mathrm{G}_{2} / \mathrm{M}$ phase arrest through inhibition of cyclinB1-Cdc2 binding. BTG2 was a direct target gene of miR-21 in the liver cancer cells. These results suggested that understanding the miR-21/BTG2 pathway may elucidate a novel regulatory mechanism for liver tumorigenesis, and provide a novel diagnostic and therapeutic target for HCC in the future.

\section{Acknowledgements}

The present study was supported by the National Natural Science Foundation of China (grant nos. 81272498, 81301631, and 30973457) and the Chongqing National Natural Science Foundation of China (grant no. cstc 2013jjB9901).

\section{References}

1. Saito Y, Hibino S and Saito H: Alterations of epigenetics and microRNA in hepatocellular carcinoma. Hepatol Res 44: 31-42, 2014.

2. Liu J, Liu X, Cui F, Chen G, Guan Y and He J: The efficacy of the inhalation of an aerosolized Group A streptococcal preparation in the treatment of lung cancer. Chin J Cancer Res 24: 346-352, 2012.

3. Skinner HD and Komaki R: Proton radiotherapy in the treatment of lung cancer. Transl Cancer Res 1: 264-270, 2012.

4. Quintavalle C and Condorelli G: Dulanermin in cancer therapy: Still much to do. Transl Lung Cancer Res 1: 158-159, 2012.

5. Yamashita S, Yamamoto H, Mimori K, Nishida N, Takahashi H, Haraguchi N, Tanaka F, Shibata K, Sekimoto M, Ishii H, et al: MicroRNA-372 is associated with poor prognosis in colorectal cancer. Oncology 82: 205-212, 2012.

6. Weiland M, Gao XH, Zhou L and Mi QS: Small RNAs have a large impact: Circulating microRNAs as biomarkers for human diseases. RNA Biol 9: 850-859, 2012.

7. Tjensvoll K, Svendsen KN, Reuben JM, Oltedal S, Gilje B, Smaaland $\mathrm{R}$ and Nordgård O: miRNA expression profiling for identification of potential breast cancer biomarkers. Biomarkers 17: 463-470, 2012.

8. Papaconstantinou I, Karakatsanis A, Gazouli M, Polymeneas G and Voros D: The role of microRNAs in liver cancer. Eur J Gastroenterol Hepatol 24: 223-228, 2012.

9. Zhang B, Pan X, Cobb GP and Anderson TA: microRNAs as oncogenes and tumor suppressors. Dev Biol 302: 1-12, 2007.

10. Iorio MV and Croce CM: MicroRNAs in cancer: Small molecules with a huge impact. J Clin Oncol 27: 5848-5856, 2009.

11. Hu QY, Jiang H, Su J and Jia YQ: MicroRNAs as biomarkers for hepatocellular carcinoma: A diagnostic meta-analysis. Clin Lab 59: 1113-1120, 2013.

12. Pass HI: Biomarkers and prognostic factors for mesothelioma. Ann Cardiothorac Surg 1: 449-456, 2012.

13. Coppola V, Musumeci M, Patrizii M, Cannistraci A, Addario A, Maugeri-Saccà M, Biffoni M, Francescangeli F, Cordenonsi M, Piccolo S, et al: BTG2 loss and miR-21 upregulation contribute to prostate cell transformation by inducing luminal markers expression and epithelial-mesenchymal transition. Oncogene 32: 1843-1853, 2013.

14. Niu J, Shi Y, Tan G, Yang CH, Fan M, Pfeffer LM and Wu ZH: DNA damage induces NF- $\kappa \mathrm{B}$-dependent microRNA-21 up-regulation and promotes breast cancer cell invasion. J Biol Chem 287: 21783-21795, 2012.

15. Zhang BG, Li JF, Yu BQ, Zhu ZG, Liu BY and Yan M: microRNA-21 promotes tumor proliferation and invasion in gastric cancer by targeting PTEN. Oncol Rep 27: 1019-1026, 2012.

16. Shibuya H, Iinuma H, Shimada R, Horiuchi A and Watanabe T: Clinicopathological and prognostic value of microRNA-21 and microRNA-155 in colorectal cancer. Oncology 79: 313-320, 2010.

17. Zhang JG, Wang JJ, Zhao F, Liu Q, Jiang K and Yang GH: MicroRNA-21 (miR-21) represses tumor suppressor PTEN and promotes growth and invasion in non-small cell lung cancer (NSCLC). Clin Chim Acta 411: 846-852, 2010.

18. Gao J, Zhang Q, Xu J, Guo L and Li X: Clinical significance of serum miR-21 in breast cancer compared with CA153 and CEA. Chin J Cancer Res 25: 743-748, 2013. 
19. Petrović N, Mandušić V, Stanojević B, Lukić S, Todorović L, Roganović J and Dimitrijević B: The difference in miR-21 expression levels between invasive and non-invasive breast cancers emphasizes its role in breast cancer invasion. Med Oncol 31: 867, 2014.

20. Li T, Leong MH, Harms B, Kennedy $G$ and Chen L: MicroRNA-21 as a potential colon and rectal cancer biomarker. World J Gastroenterol 19: 5615-5621, 2013.

21. Liu J, Zhu H, Yang X, Ge Y, Zhang C, Qin Q, Lu J, Zhan L, Cheng H and Sun X: MicroRNA-21 is a novel promising target in cancer radiation therapy. Tumour Biol 35: 3975-3979, 2014.

22. Li L, Zhou L, Li Y, Lin S and Tomuleasa C: MicroRNA-21 stimulates gastric cancer growth and invasion by inhibiting the tumor suppressor effects of programmed cell death protein 4 and phosphatase and tensin homolog. J BUON 19: 228-236, 2014

23. Liu M, Wu H, Liu T, Li Y, Wang F, Wan H, Li X and Tang H: Regulation of the cell cycle gene, BTG2, by miR-21 in human laryngeal carcinoma. Cell Res 19: 828-837, 2009.

24. Lim IK: TIS21 (/BTG2/PC3) as a link between ageing and cancer: Cell cycle regulator and endogenous cell death molecule. J Cancer Res Clin Oncol 132: 417-426, 2006.

25. Melamed J, Kernizan S and Walden PD: Expression of B-cell translocation gene 2 protein in normal human tissues. Tissue Cell 34: 28-32, 2002.

26. Wei S, Hao C, Li X, Zhao H, Chen J and Zhou Q: Effects of BTG2 on proliferation inhibition and anti-invasion in human lung cancer cells. Tumour Biol 33: 1223-1230, 2012.

27. Wagener N, Bulkescher J, Macher-Goeppinger S, Karapanagiotou-Schenkel I, Hatiboglu G, Abdel-Rahim M, Abol-Enein H, Ghoneim MA, Bastian PJ, Müller SC, et al: Endogenous BTG2 expression stimulates migration of bladder cancer cells and correlates with poor clinical prognosis for bladder cancer patients. Br J Cancer 108: 973-982, 2013.

28. Choi KS, Kim JY, Lim SK, Choi YW, Kim YH, Kang SY, Park TJ and Lim IK: TIS21(/BTG2/PC3) accelerates the repair of DNA double strand breaks by enhancing Mre11 methylation and blocking damage signal transfer to the Chk2(T68)-p53(S20) pathway. DNA Repair (Amst) 11: 965-975, 2012.

29. Zhang YJ, Wei L, Liu M, Li J, Zheng YQ, Gao Y and Li XR: BTG2 inhibits the proliferation, invasion, and apoptosis of MDA-MB-231 triple-negative breast cancer cells. Tumour Biol 34: 1605-1613, 2013.

30. Zhang Z, Chen C, Wang G, Yang Z, San J, Zheng J, Li Q, Luo X, $\mathrm{Hu}$ Q, LiZ and Wang D: Aberrant expression of the p53-inducible antiproliferative gene BTG2 in hepatocellular carcinoma is associated with overexpression of the cell cycle-related proteins. Cell Biochem Biophys 61: 83-91, 2011.
31. Zhang ZM, Wang G, Yang ZX, Shan JL, Chen C, Jin F, Xu W, Li Q, Luo XZ, Wang D and Li ZP: The expression of B-cell translocation gene 2 in diethylnitrosamine-induced primary hepatocellular carcinoma rat model. Zhonghua Gan Zang Bing Za Zhi 17: 107-111, 2009 (In Chinese).

32. Yang CH, Yue J, Pfeffer SR, Handorf CR and Pfeffer LM: MicroRNA miR-21 regulates the metastatic behavior of B16 melanoma cells. J Biol Chem 286: 39172-39178, 2011.

33. Takahashi F, Chiba N, Tajima K, Hayashida T, Shimada T, Takahashi M, Moriyama H, Brachtel E, Edelman EJ, Ramaswamy S and Maheswaran S: Breast tumor progression induced by loss of BTG2 expression is inhibited by targeted therapy with the ErbB/HER inhibitor lapatinib. Oncogene 30: 3084-3095, 2011

34. Zhang L, Huang H, Wu K, Wang M and Wu B: Impact of BTG2 expression on proliferation and invasion of gastric cancer cells in vitro. Mol Biol Rep 37: 2579-2586, 2010.

35. Livak KJ and Schmittgen TD: Analysis of relative gene expression data using real-time quantitative PCR and the 2(-Delta Delta C(T)) Method. Methods 25: 402-408, 2001

36. Krek A, Grün D, Poy MN, Wolf R, Rosenberg L, Epstein EJ, MacMenamin P, da Piedade I, Gunsalus KC, Stoffel M and Rajewsky N: Combinatorial microRNA target predictions. Nat Genet 37: 495-500, 2005.

37. Lewis BP, Shih IH, Jones-Rhoades MW, Bartel DP and Burge CB: Prediction of mammalian microRNA targets. Cell 115: 787-798, 2003.

38. Nair VS, Maeda LS and Ioannidis JP: Clinical outcome prediction by microRNAs in human cancer: A systematic review. J Natl Cancer Inst 104: 528-540, 2012.

39. Ross SA and Davis CD: MicroRNA, nutrition, and cancer prevention. Adv Nutr 2: 472-485, 2011.

40. Li Q, Wang G, Shan JL, Yang ZX, Wang HZ, Feng J, Zhen JJ, Chen C, Zhang ZM, Xu W, et al: MicroRNA-224 is upregulated in HepG2 cells and involved in cellular migration and invasion. J Gastroenterol Hepatol 25: 164-171, 2010.

41. Li Q, Ding C, Chen C, Zhang Z, Xiao H, Xie F, Lei L, Chen Y, Mao B, Jiang M, et al: miR-224 promotion of cell migration and invasion by targeting Homeobox D 10 gene in human hepatocellular carcinoma. J Gastroenterol Hepatol 29: 835-842, 2014.

42. Zhu S, Si ML, Wu H and Mo YY: MicroRNA-21 targets the tumor suppressor gene tropomyosin 1 (TPM1). J Biol Chem 282: 14328-14336, 2007.

43. Ryu MS, Lee MS, Hong JW, Hahn TR, Moon E and Lim IK: TIS21/BTG2/PC3 is expressed through PKC-delta pathway and inhibits binding of cyclin B1-Cdc2 and its activity, independent of p53 expression. Exp Cell Res 299: 159-170, 2004. 